\title{
Spatio-temporally resolved in situ transmission electron microscopy of the dynamics of nanostructured materials
}

\author{
Thomas W. Hansen ${ }^{1}$, Pei Liu ${ }^{1}$, Jacob Madsen², Philomena Schlexer ${ }^{3}$, Belá Sebök ${ }^{2}$, Jakob Schiøtz ${ }^{2}$ and \\ Jakob B. Wagner ${ }^{1}$ \\ 1. Center for Electron Nanoscopy, Technical University of Denmark, DK-2800 Kgs. Lyngby, Denmark \\ 2. Department of Physics, Technical University of Denmark, DK-2800 Kgs. Lyngby, Denmark \\ 3. Dipartimento di Scienza dei Materiali, Università degli Studi Milano-Bicocca, 20125 Milano, Italy
}

Recent advances in MEMS based instrumentation for transmission electron microscopy has paved the way for in situ investigation of materials with unprecedented spatial resolution under high pressures and elevated temperatures. Combined with traditional differentially pumped environmental TEMs, researchers can investigate phenomena such as the melting of nanoparticles, growth of individual graphene layers [1], nanowire [2] and nanotube growth and surface structure of catalytic materials exposed to pressures in the range spanning from $10^{-8} \mathrm{~Pa}$ to atmospheric pressure all at a resolution in the Ångstrøm regime. In addition, newly developed image acquisition equipment, CMOS based and direct electron detection, provides the ideal platform for analyzing the dynamics of nanostructured materials.

Given the resolution of modern electron microscopes and the precision with which atomic positions are mapped, the interplay with computational modelling has gained momentum. Here, we have modelled the melting point of platinum nanoparticles using molecular dynamics as a function of nanoparticle diameter using molecular dynamics simulations performed in the Atomic Simulation Environment (ASE) in combination with the ASAP package [3]. Experimentally we have measured the melting point in an image aberration corrected FEI Titan 80-300 TEM by monitoring the crystalline structure of the particles (see Fig. 1). The sample consisted of size selected metallic clusters deposited on a MEMS heater from DENSsolutions. The extremely low thermal drift together with the high accuracy temperature measurement provided by the MEMS based heaters allow us to accurately monitor the phase of the particles. As the temperature is increased, a disordered shell of increasing thickness was observed surrounding the particles, see Fig. 1. A good agreement between calculations and experimental observation was achieved for particles included in this study (ranging from 3-7 nm diameter).

Under the operating conditions typically found in catalytic reactors, materials tend to undergo restructuring, especially at the surface. Furthermore, the under-coordinated surface structure of the nanoparticles often used as catalyst, is not necessarily the same in nanostructured form compared to the bulk counterpart. Hence, in order gain insight into the active phase of the material, materials must be investigated in situ. Changes in particle size and structure can be quick in the beginning and more subtle as the environment equilibrates. For example, this is seen as a rapid increase in nanoparticle diameter or even phase changes in the early life of a catalyst [4]. Using HRTEM, the surface structure as a function of the surroundings was investigated. Figure 2 shows a gold particle in vacuum and exposed to oxygen, both at room temperature. Under the illumination conditions used in this investigation, the particles were stable in vacuum. However, when exposed to oxygen, columns of low coordination are observed to rapidly shift position. Using in-house developed algorithms to detect the crystal structure, the lattice constant of the outmost layers of the particles was observed to change as a function of the changing surroundings. This phenomenon was previously reported by Yoshida et al. [5]. 
Along with these results, other recent observation using the new technologies will be shown. The interaction of the electron beam with the gas phase as well as the solid will be discussed in more general terms.

\section{References:}

[1] J. Kling, T.W. Hansen, and J.B. Wagner, Carbon 99 (2016), p. 261.

[2] S. Rackauskas, et al, Nano Letters 14 (2014), p. 5810.

[3] S.R. Bahn and K.W. Jacobsen, Computing in Science \& Engineering 4 (2002), p. 56.

[4] T.W. Hansen, et al, Accounts of Chemical Research 46 (2013), p. 1720.

[5] H. Yoshida, et al, Science 335 (2012), p. 317.

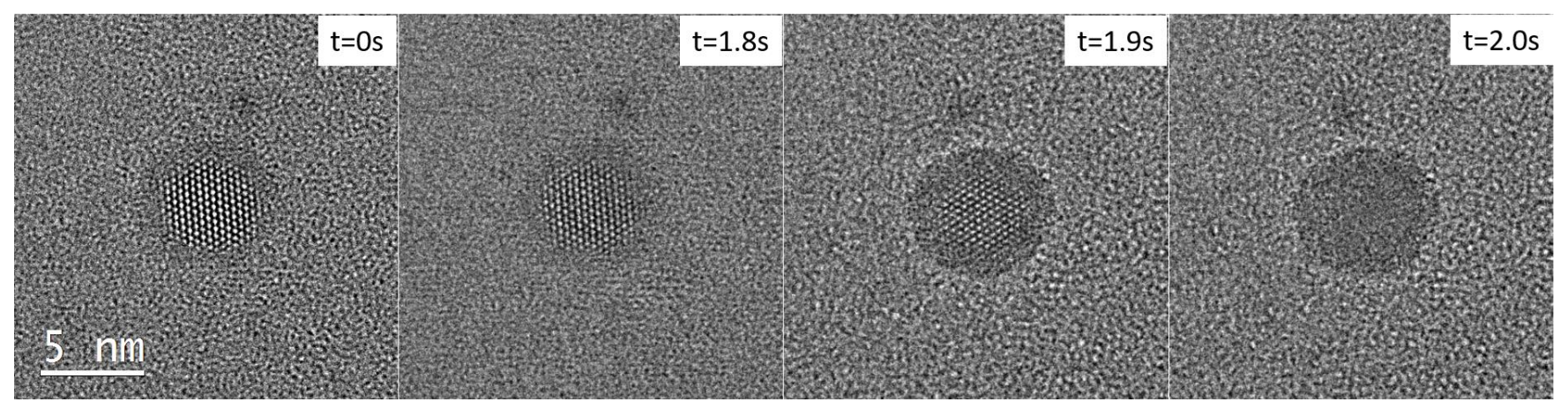

Figure 1. A $5 \mathrm{~nm}$ platinum nanoparticle imaged in vacuum at increasing temperature. The first frame is acquired at $1010^{\circ} \mathrm{C}$, subsequent frames at $1020^{\circ} \mathrm{C}$. A shell of increasing thickness is visible until the particle is completely melted.

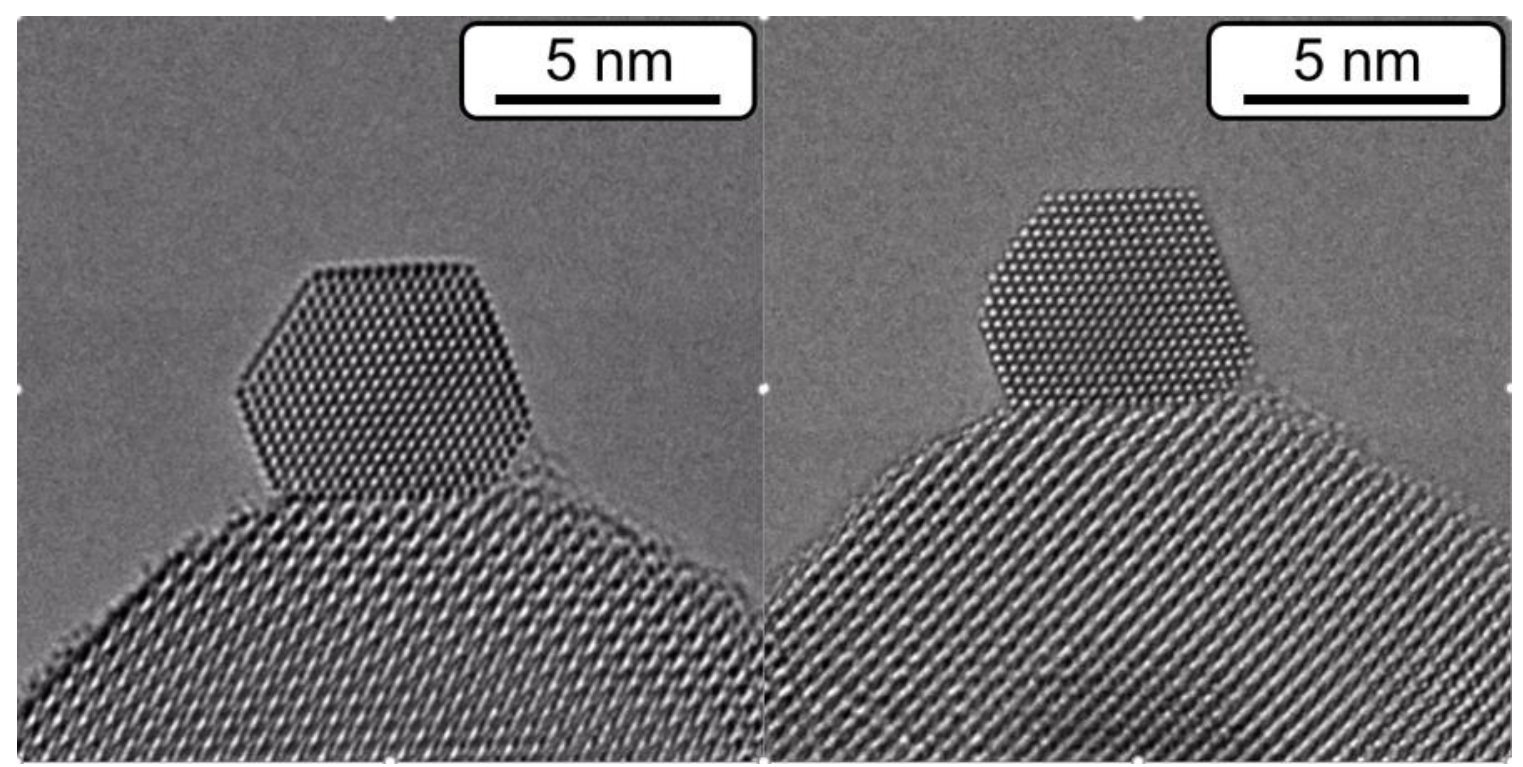

Figure 2. A $5 \mathrm{~nm}$ gold particle in vacuum (left) and exposed to $2 \mathrm{~Pa}$ oxygen (right). In oxygen atmosphere, the low coordinated sites become more mobile and columns shift position. 